\title{
Current status of top-specific variant axion model
}

\author{
Cheng-Wei Chiang, ${ }^{1,2,3,{ }^{*}}$ Hajime Fukuda, ${ }^{3, \dagger}$ Michihisa Takeuchi, ${ }^{3, *}$ and Tsutomu T. Yanagida, ${ }^{3, \S}$ \\ ${ }^{1}$ Department of Physics, National Taiwan University, Taipei, Taiwan 10617, Republic of China \\ ${ }^{2}$ Institute of Physics, Academia Sinica, Taipei, Taiwan 11529, Republic of China \\ ${ }^{3}$ Kavli IPMU (WPI), UTIAS, The University of Tokyo, Kashiwa, Chiba 277-8583, Japan
}

(Received 10 January 2018; published 26 February 2018)

\begin{abstract}
The invisible variant axion model is one of the very attractive models which solves the strong $C P$ problem but does not provoke the domain wall problem. At the electroweak scale, this model requires at least two Higgs doublets, one of which carries a nonzero Peccei-Quinn (PQ) charge and the other is neutral. We consider a scenario where only the right-handed top quark is charged under the PQ symmetry and couples with the PQ-charged Higgs doublet. As a general prediction of this model, the top quark can decay to the observed standard model-like Higgs boson $h$ and the charm or up quark, $t \rightarrow h c / u$, which recently exhibited slight excesses at LHC run-I and run-II and will soon be testable at the LHC run-II. If the rare top decay excess stays at the observed central value, we show that $\tan \beta \sim 1$ or smaller is preferred by the Higgs data. The chiral nature of the Higgs flavor-changing interaction is a distinctive feature of this model and testable using the angular distribution of the $t \rightarrow c h$ decays at the LHC.
\end{abstract}

DOI: $10.1103 /$ PhysRevD.97.035015

\section{INTRODUCTION}

The Peccei-Quinn (PQ) mechanism [1] elegantly solves the strong $C P$ problem, where a $\mathrm{U}(1)_{\mathrm{PQ}}$ symmetry is employed to rotate away $\theta_{\mathrm{QCD}}$, the $C P$-violating phase in QCD. As the PQ symmetry must be broken spontaneously, it predicts the existence of a Nambu-Goldstone boson. Since the PQ symmetry is anomalous, the additional light degree of freedom associated with the symmetry breaking becomes a massive pseudo Nambu-Goldstone boson, the axion $[2,3]$. The axion dynamics is characterized by the axion decay constant $f_{a}$, whose lower bound is obtained from axion helioscopes and astronomical observations as $f_{a} \gtrsim 10^{9} \mathrm{GeV}$ (see, for example, Ref. [4]). Moreover, coherent oscillations of the axion field can play the role of cold dark matter in the present Universe [5-7], from which we obtain the preferred range $f_{a} \sim 10^{10-12} \mathrm{GeV}[8,9]$, if the axion is the dominant component of dark matter. This beautiful mechanism, however, suffers from the domain wall formation problem in the early Universe. This is

\footnotetext{
chengwei@phys.ntu.edu.tw

†hajime.fukuda@ipmu.jp

*michihisa.takeuchi@ipmu.jp

§sutomu.tyanagida@ipmu.jp

Hamamatsu Professor.
}

Published by the American Physical Society under the terms of the Creative Commons Attribution 4.0 International license. Further distribution of this work must maintain attribution to the author(s) and the published article's title, journal citation, and DOI. Funded by SCOAP ${ }^{3}$. because the model has $N_{D W}=3$ discrete vacua directly related to the number of fermion families.

The variant axion model introduced in Refs. [10,11] is one of the interesting axion models free from the domain wall problem. In this model, it is solved by allowing only one right-handed quark to carry a PQ charge and thus rendering a unique vacuum $\left(N_{\mathrm{DW}}=1\right)$ [12]. The model requires two Higgs doublet fields for consistency, where one of which is charged under the PQ symmetry. As a result, there is a nontrivial flavor structure in the Yukawa couplings [13] that can lead to the flavor-changing neutral-current (FCNC) couplings of the Higgs bosons to at least quarks. Moreover, in this model we see the characteristic charity nature of these FCNC couplings, which is not seen in the common two Higgs doublet models (2HDMs). Therefore, the variant axion model exhibits interesting and distinctive phenomena in flavor physics at low energies.

In Refs. [13,14], we had considered such a 2HDM with the PQ symmetry and assigned a nonzero charge to the righthanded top quark, thus dubbed the top-specific variant axion model. Previously, we had performed the parameter scan based on the constraints on the mixing parameters in the Higgs sector using the LHC run-I data [14]. In particular, there was an interesting excess in the $h \rightarrow \tau \mu$ decay $[15,16]$ at that time. Therefore, we had focused on the compatibility of this model to the excess and discussed the connection between the quark sector and the lepton sector. Recently, the new run-II results negate the excess and have a result being well consistent with the standard model (SM) prediction [17]. In this letter, we aim to update the parameter fitting using the current Higgs data and study its primary 
signature of the $t \rightarrow h c / u$ decays that are being probed at the LHC.

Recently, the ATLAS Collaboration has reported a slight excess in the $t \rightarrow c h$ channel at $1.5 \sigma$ level, $\mathrm{BR}(t \rightarrow c h)=$ $(0.22 \pm 0.14) \%$, using the full set of the LHC run-I data [18]. That excess is consistent with the corresponding CMS observation at LHC run-I, given by $\operatorname{BR}(t \rightarrow c h)<0.40 \%$ [or $\mathrm{BR}(t \rightarrow u h)<0.55 \%$ ] at 95\% confidence level (C.L.) [19]. More recently, an ATLAS analysis based on the LHC run-II data is published and also suggests an interesting excess in the $t \rightarrow c h$ decay [20], where the observed results set an upper bound $\operatorname{BR}(t \rightarrow c h)<0.22 \%$ at $95 \%$ C.L., while the expected one is $\operatorname{BR}(t \rightarrow c h)<0.16 \%$, which corresponds to a $1 \sigma$ excess. Although currently the data seem to merely show a small upper fluctuation, it may turn out to be revealing a real new physics signature in the long run. It is thus worth discussing whether the variant axion model is compatible with such an excess at this point, since such a FCNC top decay is one of the robust and distinctive predictions of the model.

In this paper, we take the central value of $\mathrm{BR}(t \rightarrow c h)=$ $0.22 \%$ given by the ATLAS in run-I as a nominal value (also the 95\% C.L. upper bound of the ATLAS run-II determination). It corresponds to $\lambda_{t c H}=0.090$ or $a^{2} \sin ^{2} \rho=$ $\left(2.2 \times 10^{-3}\right) /\left(3.24 \times 10^{-2}\right)=0.068$ in our model parameters. To obtain such a sizable effect, large mixing in $\rho$ or large $a=\cos (\beta-\alpha)(\tan \beta+\cot \beta)$ is required. Therefore, the off-diagonal couplings have to be sizable and it requires a careful check whether the parameter space is compatible with perturbativity conditions. By taking the current SM-like Higgs data into account, we set constraints on the parameter space of the model. We find that all the constraints are satisfied when $\tan \beta \sim 1$. Especially, $\tan \beta<1$ is preferred in order to have sizable top FCNC effects without having conflicts with the other Higgs observables and theoretical restrictions. We also show how the chirality nature in the FCNCs can be probed by studying the angular distribution of the $t \rightarrow c h$ decay at LHC run-II.

A crude estimate for the future sensitivity on the $t \rightarrow c h$ decay without optimization was given as $2 \times 10^{-3}$ $\left(5 \times 10^{-4}\right)$ in the lepton channels and $5 \times 10^{-4}\left(2 \times 10^{-4}\right)$ in the photon channels [21-23], assuming an integrated luminosity of $300 \mathrm{fb}^{-1}\left(3 \mathrm{ab}^{-1}\right)$ in run-III. In fact, one notes that the current sensitivity already approaches a similar level by combining the $\gamma \gamma$, multilepton, and $b b$ modes, with just the LHC run-I ( 7 and $8 \mathrm{TeV}$ ) data of $\mathcal{O}(25) \mathrm{fb}^{-1}$. We can expect the final combined sensitivity at $3 \mathrm{ab}^{-1}$ to reach below $0.01 \%$.

This paper is organized as follows. Section II discusses the structure of the Higgs sector along with the FCNC couplings of the SM-like Higgs boson to fermions in the top-specific variant axion model. We also discuss the expected sizes of FCNC decays and theoretical constraints on this model. In Sec. III, we perform a $\chi^{2}$-fit analysis based on the latest Higgs signal strength data at the LHC. In Sec. IV, we discuss how to confirm this model through the angular distribution of $t \rightarrow c h$ decay and show the expected sensitivity corresponding to the scenario considered in this work. Conclusions are given in Sec. V.

\section{TOP-SPECIFIC VARIANT AXION MODEL}

In the variant axion model, we introduce two Higgs doublet fields $\Phi_{1}$ and $\Phi_{2}$ and a scalar field $\sigma$ with PQ charges $0,-1$ and 1 , respectively as a minimal setup. The gauge singlet scalar $\sigma$ acquires a vacuum expectation value (VEV) $f_{a}$ at a high-energy scale, which breaks the PQ symmetry spontaneously although it does not play much of a role at low energies. In the quark sector, we assume that only the right-handed top quark field $t_{R}$ possesses a nonzero PQ charge of -1 . Note that we can additionally assign nonzero PQ charges to leptons as well, as they do not contribute to the number of domain walls $N_{\mathrm{DW}}$ [12]. In this paper, we focus on the scenario where the leptons have no PQ charges. The interesting phenomenology of the case when the leptons also carry PQ charges can be found in our previous analysis [14].

The most general renormalizable Higgs potential obeying the PQ symmetry with the above PQ charge assignments is, as already given in Refs. [13,14,24],

$$
\begin{aligned}
V\left(\Phi_{1}, \Phi_{2}\right)= & m_{11}^{2} \Phi_{1}^{\dagger} \Phi_{1}+m_{22}^{2} \Phi_{2}^{\dagger} \Phi_{2}-\left(m_{12}^{2} \Phi_{1}^{\dagger} \Phi_{2}+\text { H.c. }\right) \\
& +\frac{\lambda_{1}}{2}\left(\Phi_{1}^{\dagger} \Phi_{1}\right)^{2}+\frac{\lambda_{2}}{2}\left(\Phi_{2}^{\dagger} \Phi_{2}\right)^{2} \\
& +\lambda_{3}\left(\Phi_{1}^{\dagger} \Phi_{1}\right)\left(\Phi_{2}^{\dagger} \Phi_{2}\right)+\lambda_{4}\left(\Phi_{1}^{\dagger} \Phi_{2}\right)\left(\Phi_{2}^{\dagger} \Phi_{1}\right),
\end{aligned}
$$

where the $\sigma$ field has been integrated out. The $m_{12}^{2}$ terms softly violate the PQ symmetry (as can be derived from a UV-complete Lagrangian [13]), and can be made real and positive through a rotation of the PQ symmetry. All the other terms respect the PQ symmetry and their associated parameters $\left(m_{11}^{2}, m_{22}^{2}\right.$, and $\left.\lambda_{1,2,3,4}\right)$ are real.

After the electroweak symmetry breaking, each $\Phi_{i}$ acquires a respective VEV $v_{i}$ and can be written in terms of component fields as $\Phi_{i}=\left(H_{i}^{+},\left(v_{i}+h_{i}+i A_{i}\right) / \sqrt{2}\right)^{T}$. We define $\tan \beta=v_{2} / v_{1}$ as usual and $v_{\mathrm{SM}}^{2}=v_{1}^{2}+v_{2}^{2} \simeq$ $(246 \mathrm{GeV})^{2}$. We now rotate the Higgs doublets to the so-called Higgs basis [25], where only one of the doublets $\left(\Phi^{\mathrm{SM}}\right)$ has a nonzero VEV,

$$
\begin{aligned}
\left(\begin{array}{c}
\Phi_{1} \\
\Phi_{2}
\end{array}\right) & =R_{\beta}\left(\begin{array}{c}
\Phi^{\mathrm{SM}} \\
\Phi^{\prime}
\end{array}\right), \quad \text { with } \\
R_{\theta} & =\left(\begin{array}{cc}
\cos \theta & -\sin \theta \\
\sin \theta & \cos \theta
\end{array}\right), \\
\text { and } \quad \Phi^{\mathrm{SM}} & =\left(\begin{array}{c}
G^{+} \\
\left(v_{\mathrm{SM}}+h^{\mathrm{SM}}+i G^{0}\right) / \sqrt{2}
\end{array}\right), \\
\Phi^{\prime} & =\left(\begin{array}{c}
H^{+} \\
\left(h^{\prime}+i A^{0}\right) / \sqrt{2}
\end{array}\right),
\end{aligned}
$$


where $G^{ \pm}$and $G^{0}$ are the would-be Nambu-Goldstone bosons to become the longitudinal modes of the $W^{ \pm}$and $Z$ bosons. The pseudoscalar Higgs boson $A^{0}$ and charged Higgs boson $H^{ \pm}$are mass eigenstates with masses $m_{A}$ and $m_{H^{+}}$, respectively. We also define the mass eigenstates of the $C P$-even neutral Higgs bosons as $h$ and $H$, with respective masses $m_{h}$ and $m_{H}\left(m_{h}<m_{H}\right)$, through a rotation of angle $\alpha$ from the original PQ basis as follows:

$$
\left(\begin{array}{c}
H \\
h
\end{array}\right)=R_{-\alpha}\left(\begin{array}{l}
h_{1} \\
h_{2}
\end{array}\right)=R_{\beta-\alpha}\left(\begin{array}{c}
h^{\mathrm{SM}} \\
h^{\prime}
\end{array}\right) .
$$

Note that the light Higgs boson $h$ becomes a SM-like Higgs boson $h^{\mathrm{SM}}$ in the limit of $\sin (\beta-\alpha) \rightarrow 1$, which can be realized when $m_{12}^{2} \rightarrow \infty$. The couplings between $h$ and weak gauge bosons are read as

$g_{h V V}=\sin (\beta-\alpha) g_{h V V}^{\mathrm{SM}}, \quad g_{H V V}=\cos (\beta-\alpha) g_{h V V}^{\mathrm{SM}}, \quad$ and

$g_{A V V}=0$,

where $g_{h V V}^{\mathrm{SM}}$ are the couplings in the SM. The triple Higgs coupling $\lambda_{h H^{+} H^{-}}$, defined by the $\lambda_{h H^{+} H^{-}} h H^{+} H^{-}$interaction term in the Lagrangian, is given by

$$
\begin{aligned}
\lambda_{h H^{+} H^{-}}= & \frac{1}{v_{\mathrm{SM}}}\left[\left(m_{h}^{2}+2 m_{H^{+}}^{2}-2 m_{A}^{2}\right) \sin (\beta-\alpha)\right. \\
& \left.+\left(m_{A}^{2}+m_{h}^{2}\right)(\tan \beta-\cot \beta) \cos (\beta-\alpha)\right],
\end{aligned}
$$

where $m_{A}^{2} \equiv 2 m_{12}^{2} / \sin 2 \beta$.

For Yukawa interactions, since we consider the scenario in which only the right-handed top quark carries a nonzero PQ charge among all quark fields, the up-type Yukawa interaction Lagrangian is

$$
L^{u}=-\Phi_{1} \bar{u}_{R a}\left[Y_{u 1}\right]_{a i} q_{i}-\Phi_{2} \bar{u}_{R 3}\left[Y_{u 2}\right]_{3 i} q_{i}+\text { H.c. },
$$

where the family indices $a=1,2$ and $i, j=1,2,3$. Schematically, the Yukawa coupling matrices, $Y_{u 1}$ and $Y_{u 2}$, in the original PQ basis take the forms

$$
Y_{u 1}=\left(\begin{array}{ccc}
* & * & * \\
* & * & * \\
0 & 0 & 0
\end{array}\right), \quad Y_{u 2}=\left(\begin{array}{ccc}
0 & 0 & 0 \\
0 & 0 & 0 \\
* & * & *
\end{array}\right),
$$

where $*$ indicates a generally nonzero element.

In the Higgs basis, the up-type Yukawa interaction Lagrangian can be expressed as

$$
L^{u}=-\Phi^{\mathrm{SM}} \bar{u}_{R i}\left[Y_{u}^{\mathrm{SM}}\right]_{i j} q_{j}-\Phi^{\prime} \bar{u}_{R i}\left[Y_{u}^{\prime}\right]_{i j} q_{j}+\text { H.c. },
$$

and the Yukawa matrices are

$$
\begin{aligned}
Y_{u}^{\mathrm{SM}} & =\cos \beta Y_{u 1}+\sin \beta Y_{u 2}, \\
Y_{u}^{\prime} & =-\sin \beta Y_{u 1}+\cos \beta Y_{u 2} \\
& =\left(\begin{array}{rrr}
-\tan \beta & & \\
& -\tan \beta & \\
& & \cot \beta
\end{array}\right) Y_{u}^{\mathrm{SM}} .
\end{aligned}
$$

Therefore, the number of degrees of freedom in the up-type Yukawa matrices of this model is the same as that of the SM. At this stage, the mass matrix $M_{u} \equiv \frac{v_{\mathrm{SM}}}{\sqrt{2}} Y_{u}^{\mathrm{SM}}$ is generally nondiagonal and can be brought to its diagonal form through a biunitary transformation $V M_{u} U^{\dagger}=$ $\operatorname{diag}\left(m_{u}, m_{c}, m_{t}\right) \equiv \frac{v_{\mathrm{SM}}}{\sqrt{2}} Y_{u}^{\mathrm{diag}}$, where $U$ and $V$ are two unitary matrices, which rotates the left-handed fields $q_{i}$ and the right-handed fields $u_{R, i}$, respectively. In this basis, the other Yukawa matrix becomes

$$
\begin{aligned}
Y_{u}^{\prime \text { diag }}= & \left(\begin{array}{lll}
-\tan \beta & & \\
& -\tan \beta & \\
& & \cot \beta
\end{array}\right) Y_{u}^{\mathrm{diag}} \\
& +(\tan \beta+\cot \beta) H_{u} Y_{u}^{\mathrm{diag}},
\end{aligned}
$$

where the Hermitian matrix

$$
H_{u} \equiv V\left(\begin{array}{ccc}
0 & & \\
& 0 & \\
& & 1
\end{array}\right) V^{\dagger}-\left(\begin{array}{ccc}
0 & & \\
& 0 & \\
& & 1
\end{array}\right) .
$$

Note that in the second term of Eq. (10), the $(\tan \beta+$ $\cot \beta) H_{u}$ part describes mixing among up-type quarks and $Y_{u}^{\text {diag }}$ controls the strength of coupling with the dominant component given by the top Yukawa coupling. For simplicity, we will omit the superscript "diag" while working in the mass-diagonal basis in the following discussions. Note that $V$ is the rotation matrix for the right-handed up-type quarks and is completely independent of the CabibboKobayashi-Maskawa matrix, which is the product of lefthanded up-type and left-handed down-type quark rotation matrices. Therefore, the mixing angles in $V$ can be as large as $\mathcal{O}(1)$, a key intriguing feature of the model.

As an illustration and in anticipation of interesting collider phenomenology associated with the top quark, we restrict ourselves to the case of $t-c$ mixing in this paper as in Ref. [14]. In such a simplified scenario without introducing new $C P$ phases, the mixing matrices $H_{u}$ and $V$ can be parametrized in terms of $\rho$ only as

$$
\begin{gathered}
H_{u}=\left(\begin{array}{ccc}
0 & 0 & 0 \\
0 & \frac{1-\cos \rho}{2} & \frac{\sin \rho}{2} \\
0 & \frac{\sin \rho}{2} & \frac{\cos \rho-1}{2}
\end{array}\right), \\
V=\left(\begin{array}{ccc}
1 & 0 & 0 \\
0 & \cos \frac{\rho}{2} & \sin \frac{\rho}{2} \\
0 & -\sin \frac{\rho}{2} & \cos \frac{\rho}{2}
\end{array}\right),
\end{gathered}
$$


and the Yukawa interactions of the observed Higgs boson $h$ in the mass eigenbasis are then described by

$$
\begin{gathered}
\mathcal{L}_{Y} \equiv-\sum_{f=e, \ldots, u, \ldots, d, \cdots} \xi_{f} \frac{m_{f}}{v_{\mathrm{SM}}} h \bar{f} f+\mathcal{L}_{\mathrm{FCNC}} \\
\text { with } \quad \mathcal{L}_{\mathrm{FCNC}}=\mathcal{L}_{t c}=-\frac{a \sin \rho}{2 v_{\mathrm{SM}}}\left(m_{t} \bar{c}_{R} t_{\mathcal{L}}+m_{c} \bar{t}_{R} c_{\mathcal{L}}\right) h+\text { H.c. }
\end{gathered}
$$

where $a \equiv(\tan \beta+\cot \beta) \cos (\beta-\alpha)$ and

$$
\xi_{f}= \begin{cases}\sin (\beta-\alpha)+\left(\cot \beta-\frac{1-\cos \rho}{2}(\tan \beta+\cot \beta)\right) \cos (\beta-\alpha) & \text { for } f=t, \\ \sin (\beta-\alpha)-\left(\tan \beta-\frac{1-\cos \rho}{2}(\tan \beta+\cot \beta)\right) \cos (\beta-\alpha) & \text { for } f=c, \\ \sin (\beta-\alpha)-\tan \beta \cos (\beta-\alpha) & \text { otherwise. }\end{cases}
$$

One striking feature of $\mathcal{L}_{\mathrm{FCNC}}$ is that the predicted flavor violation is associated with large asymmetries in the chirality. In this simplified case, the top decay is dominated by the right-handed charm-associated processes to be discussed in more detail in the next section.

\section{A. Rare top quark FCNC decay}

This model generically predicts the top FCNC decay $t \rightarrow c h$ (or $t \rightarrow u h$ ) via the mixing effect. Therefore, such decays serve as a smoking gun signature of the model. For definiteness, we focus on the $t \rightarrow c h$ decay in this section, but note that the current experimental limits do not actively tag the flavor of the accompanied jet and that what is constrained is the weighted sum of all branching ratios of $t \rightarrow q h(q=u, c)$.

The partial decay width of $t \rightarrow c h$ is given by

$$
\Gamma_{t \rightarrow c h}=\frac{G_{F} m_{t}^{3} a^{2} \sin ^{2} \rho}{64 \pi \sqrt{2}}\left(1-r_{h}^{2}\right)^{2},
$$

with $r_{h}^{2} \equiv m_{h}^{2} / m_{t}^{2} \sim 0.522$ for $m_{h}=125 \mathrm{GeV}$ and $m_{t}=$ $173 \mathrm{GeV}$. By comparing it with the width of $t \rightarrow b W$ in the SM at the leading order,

$$
\Gamma_{t \rightarrow b W}=\frac{G_{F}\left|V_{t b}\right|^{2} m_{t}^{3}}{8 \pi \sqrt{2}}\left(1-r_{W}^{2}\right)^{2}\left(1+2 r_{W}^{2}\right),
$$

with $r_{W}^{2} \equiv m_{W}^{2} / m_{t}^{2} \simeq 0.214$ for $m_{W}=80.4 \mathrm{GeV}$, we can obtain by assuming $\mathrm{BR}(t \rightarrow b W)$ is close to unity that

$$
\mathrm{BR}(t \rightarrow c h) \simeq \frac{\Gamma_{t \rightarrow c h}}{\Gamma_{t \rightarrow b W}} \simeq\left(3.24 \times 10^{-2}\right) a^{2} \sin ^{2} \rho .
$$

The nominal branching ratio of $0.22 \%$ corresponds to the mixing parameters satisfying

$$
a^{2} \sin ^{2} \rho=0.068
$$

As the future sensitivity of $0.02 \%$ for $\mathrm{BR}(t \rightarrow c h$ ) (for $14 \mathrm{TeV}$ and $3000 \mathrm{fb}^{-1}$ ) corresponds to

$$
a^{2} \sin ^{2} \rho=6.2 \times 10^{-3},
$$

the current nominal value will be fully confirmed at $5 \sigma$ level by then if the model is correct. These two values will be depicted in the following figures in pink and red, respectively.

It is noted that the $h-t-c / u$ couplings can also contribute to other flavor observables. For example, $D^{0}-\bar{D}^{0}$ meson mixing measurements constrain the products $\left|\lambda_{t u} \lambda_{t c}\right|,\left|\lambda_{u t} \lambda_{c t}\right|<7.6 \times 10^{-3},\left|\lambda_{t u} \lambda_{c t}\right|,\left|\lambda_{u t} \lambda_{t c}\right|<2.2 \times 10^{-3}$ and $\left|\lambda_{t u} \lambda_{u t} \lambda_{c t} \lambda_{t c}\right|^{1 / 2}<0.9 \times 10^{-3}[26,27]$. As we are mainly concerned with the mixing between the last two generations, combining with $\left|\lambda_{c t}\right|=\left|a \frac{m_{t}}{v_{\mathrm{SM}}}\left(H_{u}\right)_{c t}\right|=0.09$ coming from the nominal value of $\operatorname{BR}(t \rightarrow h c)$, the most stringent constraint is $\left|\lambda_{u t} \lambda_{c t}\right|=\left|a^{2} \frac{m_{t}^{2}}{v_{\mathrm{SM}}^{2}}\left(H_{u}\right)_{u t}\left(H_{u}\right)_{c t}\right| \leq$ $0.0086\left|\left(H_{u}\right)_{u t} /\left(H_{u}\right)_{c t}\right|$. Thus, the condition $\mid\left(H_{u}\right)_{u t} t$ $\left(H_{u}\right)_{c t} \mid \lesssim 0.9$ is sufficient to avoid the above-mentioned constraints while reproducing the nominal value with no significant fine tuning in the model parameters. While introducing no new $C P$-violating sources in this model, we note in passing that the imaginary parts of the flavorviolating Yukawa couplings can be constrained by the hadron electric dipole moments and $C P$-violating observables in the $D$ mesons [28].

\section{B. Perturbativity constraints}

Since $Y_{u}^{\prime, \text { diag }}$ involves $\rho$ and $\tan \beta$, some element may become too large for some sizable $\rho, \tan \beta$ or $\cot \beta$. Large Yukawa couplings could have the problem of blowing up after running to high energies. Since our model assumes the PQ symmetry to solve the strong $C P$ problem, the coupling must not blow up at least up to the PQ scale. More stringently, we require that the theory does not contain any divergent coupling up to the Planck scale. As the Yukawa 


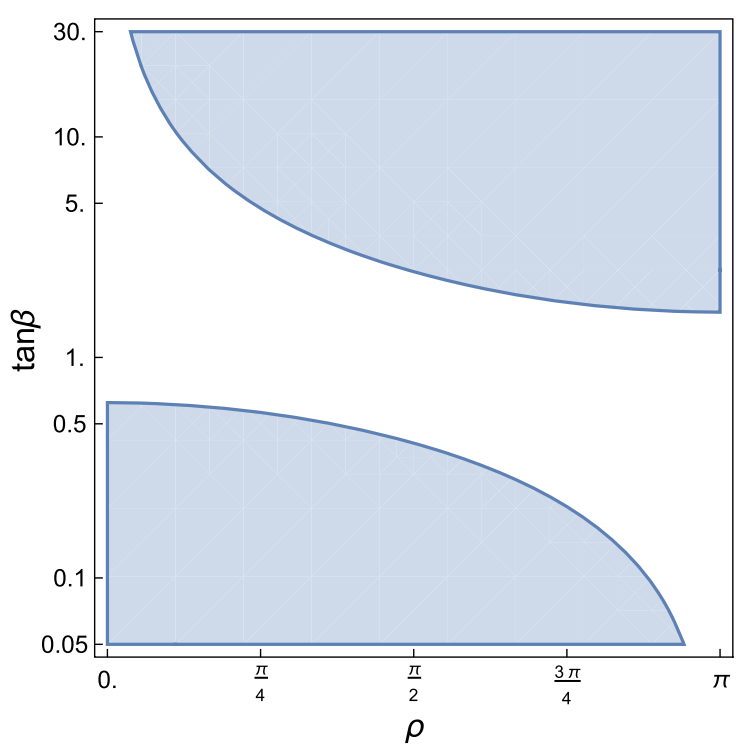

FIG. 1. The perturbative parameter space in $\rho-\tan \beta$ plane. Shaded regions are excluded by the perturbativity condition $\left(\forall i, j Y_{i j}(\mu)<4 \pi\right.$ at any $\left.\mu<M_{\text {Planck }}\right)$.

couplings are base dependent, we require that the absolute value of any Yukawa coupling is smaller than $4 \pi$ for the validity of perturbation.

We use the 1-loop renormalization equations in Eqs. (404)-(409) of Ref. [29], ignoring the Higgs self couplings, for numerical evaluations. The parameter region satisfying the above perturbative condition is shown in Fig. 1, with the excluded region shown in gray. The result can be understood as follows. For $\rho=0,\left(Y_{u}^{\prime \text {,diag }}\right)_{t t} \simeq$ $y_{t} \cot \beta$ blows up when $\tan \beta$ is small. On the other hand, for $\rho \sim \pi,\left(Y_{u}^{\prime, \text { diag }}\right)_{t t} \simeq y_{t} \tan \beta$ becomes large when $\tan \beta$ is large. For the large mixing angle region, $\rho \sim \pi / 2$, both large and small $\tan \beta$ cause the coupling to blow up, due to the large off-diagonal element

$$
\left(Y_{u}^{\prime, \text { diag }}\right)_{c t}=(\tan \beta+\cot \beta) \frac{\sin \rho}{\sqrt{2}} \frac{m_{t}}{v} .
$$

Therefore, a moderate $\tan \beta \sim 1$ is preferred by the perturbativity for a large mixing of $\rho \sim \pi / 2$. Constraints for several fixed values of $\rho$ are given as follows: $\tan \beta>0.62$ $(\rho=0), \quad 0.41<\tan \beta<2.45 \quad(\rho=\pi / 2), \quad 0.21<\tan \beta<$ $1.78(\rho=3 \pi / 4)$, and $\tan \beta<1.60(\rho=\pi)$. Note that when $\rho$ takes a finite value, the lower bound on $\tan \beta$ is relaxed compared with the usual $\rho=0$ case. When we fix $\tan \beta$ to a few values, the constraints are $1.15<\rho(\tan \beta=0.5) \rho<$ $1.99(\tan \beta=2), \rho<0.75 \quad(\tan \beta=5)$, and $\rho<0.37$ $(\tan \beta=10)$. We find that there are no constraints on $\rho$ for $0.62<\tan \beta<1.6$.

\section{HIGGS SIGNAL STRENGTH CONSTRAINTS FROM LHC RUN-II}

As we assume that the exotic Higgs bosons are sufficiently heavy to decouple from the low-energy phenomenology [14], our model is essentially parametrized by only three parameters $\alpha, \beta$ and $\rho$. In this section, we show the constraints on these model parameters using the latest LHC Higgs data. As noted earlier, the couplings

TABLE I. Higgs signal strengths of various modes measured at the LHC in 7-, 8-, and 13-TeV collisions. In the first column, ggF, $\mathrm{VBF}, \mathrm{VH}, t t H$ refers to the Higgs production mechanisms of gluon-gluon fusion, vector boson fusion, associated production with gauge bosons $(W / Z)$, and associated production with $t \bar{t}$, respectively. The superscript indicates the colliding energy, and the subscript indicates the collaboration. The first row indicates the final states of the Higgs boson. Unless quoted explicitly, the data are generally transcribed from Table 8 of Ref. [30]. In the $t t H$ rows, the numbers shown in $W W$ column is based on the analysis in the multilepton mode, where all $Z Z / W W / \tau \tau$ modes contribute although dominated by $W W$ mode. Thus, $(W W)$ means that $Z Z / \tau \tau$ modes are in principle constrained by them, while not considered in our analysis.

\begin{tabular}{|c|c|c|c|c|c|}
\hline & $\gamma \gamma$ & $Z Z$ & $W W$ & $\tau \tau$ & $b b$ \\
\hline $\operatorname{ggF}^{7,8} \mathrm{TeV}$ & $1.10_{-0.22}^{+0.23}$ & $1.13_{-0.31}^{+0.34}$ & $0.84 \pm 0.17$ & $1.0 \pm 0.6$ & $\cdots$ \\
\hline $\operatorname{ggF}_{\text {ATLAS }}^{13 \mathrm{TeV}}$ & $0.8_{-0.18}^{+0.19}[31]$ & $1.11 \pm 0.245[32]$ & $\cdots$ & $\ldots$ & $\cdots$ \\
\hline $\operatorname{ggF}_{\mathrm{CMS}}^{13 \mathrm{TeV}}$ & $1.11_{-0.18}^{+0.19}[33]$ & $1.20_{-0.21}^{+0.22}[34]$ & $0.9_{-0.3}^{+0.4}[35]$ & $1.17_{-0.40}^{+0.47}[36]$ & $2.3_{-1.6}^{+1.8}[37]$ \\
\hline $\mathrm{VBF}^{7,8} \mathrm{TeV}$ & $1.3 \pm 0.5$ & $0.1_{-0.6}^{+1.1}$ & $1.2 \pm 0.4$ & $1.3 \pm 0.4$ & $\cdots$ \\
\hline $\mathrm{VBF}_{\mathrm{ATLAS}}^{13 \mathrm{TeV}}$ & $2.1 \pm 0.6[31]$ & $4.0 \pm 1.77[32]$ & $3.2_{-4.2}^{+4.4}[38]$ & $\cdots$ & $-3.9_{-2.7}^{+2.8}[39]$ \\
\hline $\mathrm{VBF}_{\mathrm{CMS}}^{13 \mathrm{TeV}}$ & $0.54_{-0.5}^{+0.6}[33]$ & $0.06_{-0.06}^{+1.03}[34]$ & $1.4 \pm 0.8[35]$ & $1.11_{-0.35}^{+0.34}[36]$ & $-3.7_{-2.5}^{+2.4}[40]$ \\
\hline $\mathrm{VH}^{7,8} \mathrm{TeV}$ & $0.5 \pm 1.1$ & $\cdots$ & $2.3 \pm 1.0$ & $-0.2 \pm 1.1$ & $0.63 \pm 0.3$ \\
\hline $\mathrm{VH}_{\mathrm{ATLAS}}^{13 \mathrm{TeV}}$ & $0.7_{-0.8}^{+0.9}[31]$ & $<3.8[32]$ & $1.7_{-0.9}^{+1.1}[38]$ & $\cdots$ & $1.20_{-0.36}^{+0.42}[41]$ \\
\hline $\mathrm{VH}_{\mathrm{CMS}}^{13 \mathrm{TeV}}$ & $2.29_{-1.0}^{+1.1}[33]$ & $<2.8[34]$ & $-0.3 \pm 1.3[35]$ & .. & $\ldots$ \\
\hline$t t H^{7,8 \mathrm{TeV}}$ & $2.2_{-1.3}^{+1.6}$ & $\cdots$ & $5.0_{-1.7}^{+1.8}$ & $-1.9_{-3.3}^{+3.7}$ & $1.1 \pm 1.0$ \\
\hline$t t H_{\text {ATLAS }}^{13 \mathrm{TeV}}$ & $0.5 \pm 0.6[31]$ & $(W W)$ & $2.5_{-1.1}^{+1.3}[42]$ & $(W W)$ & $2.1_{-0.9}^{+1.0}[42]$ \\
\hline$t t H_{\mathrm{CMS}}^{13 \mathrm{TeV}}$ & $2.22_{-0.8}^{+0.9}[33]$ & $(W W)$ & $1.5 \pm 0.5[43]$ & $0.72_{-0.53}^{+0.62}[44]$ & $-0.19 \pm 0.80[45]$ \\
\hline
\end{tabular}



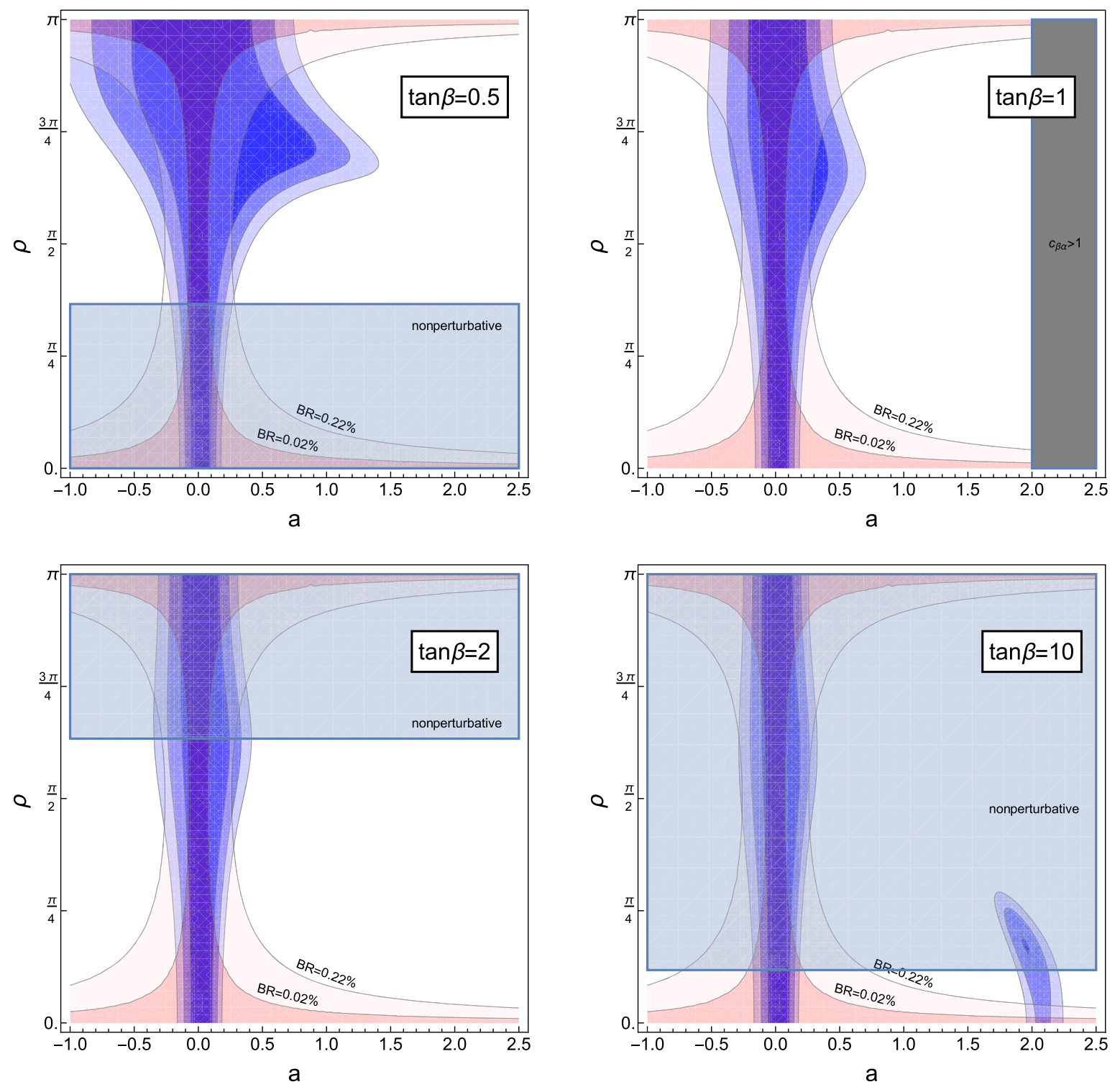

FIG. 2. Allowed parameter space at $68 \%$ (dark blue), $95 \%$ (blue) and $99 \%$ (light blue) C.L. from the latest Higgs signal strength data in the $a$ - $\rho$ plane for different values of $\tan \beta: 0.5$ (upper-left plot), 1 (upper-right plot), 2 (lower-left plot), and 10 (lower-right plot). The red contours are drawn for $\mathrm{BR}(t \rightarrow c h)=0.22 \%$ and for the conservative ultimate sensitivity of $0.02 \%$. Assuming the nominal value $0.22 \%$ for $\operatorname{BR}(t \rightarrow c h), \tan \beta$ of $\mathcal{O}(1)$ offers a broader parameter space consistent with the current Higgs data at the $1 \sigma$ level, although all the $\tan \beta$ value can be made compatible within $2 \sigma$.

between the SM-like Higgs boson $h$ and the SM particles are modified from their SM values: Eq. (4) for the gauge bosons and Eq. (15) for the fermions. We use them to estimate the signal strengths of various Higgs production channels. We note that the diphoton decay width depends to some extent on the coupling $\lambda_{h H^{+} H^{-}}$, which in turn would modify the predicted Higgs signal strengths. However, such a dependence diminishes under our assumption of heavy exotic Higgs bosons. Therefore, for definiteness, we set $\lambda_{h H^{+} H^{-}}=0$ in the following analysis.

In our global $\chi^{2}$ fit, we take all the signal strengths listed in Table I with the both-side errors (44 data) into account. They include the run-I ( 7 and $8 \mathrm{TeV}$ ) data reported by the ATLAS and CMS Collaborations summarized in Ref. [30] and the latest results from run II at $13 \mathrm{TeV}$ collected from Refs. [31-45]. In the $\chi^{2}$ function, we add the statistical and the systematic errors in quadrature and average asymmetric errors for simplicity.

In Fig. 2, we show the allowed parameter space in the $(a, \rho)$ plane for $\tan \beta$ fixed to $0.5,1,2$ and 10 . The darker blue, blue, and lighter blue regions correspond respectively to the $1 \sigma, 2 \sigma$ and $3 \sigma$ regions, based on $\Delta \chi^{2}=\chi^{2}-\chi_{\min }^{2}=$ $(3.53,8.02,14.2)$ for 3 degrees of freedom. We found $\chi_{\min }^{2}=48.3$ being an appropriate goodness of fit for 44 

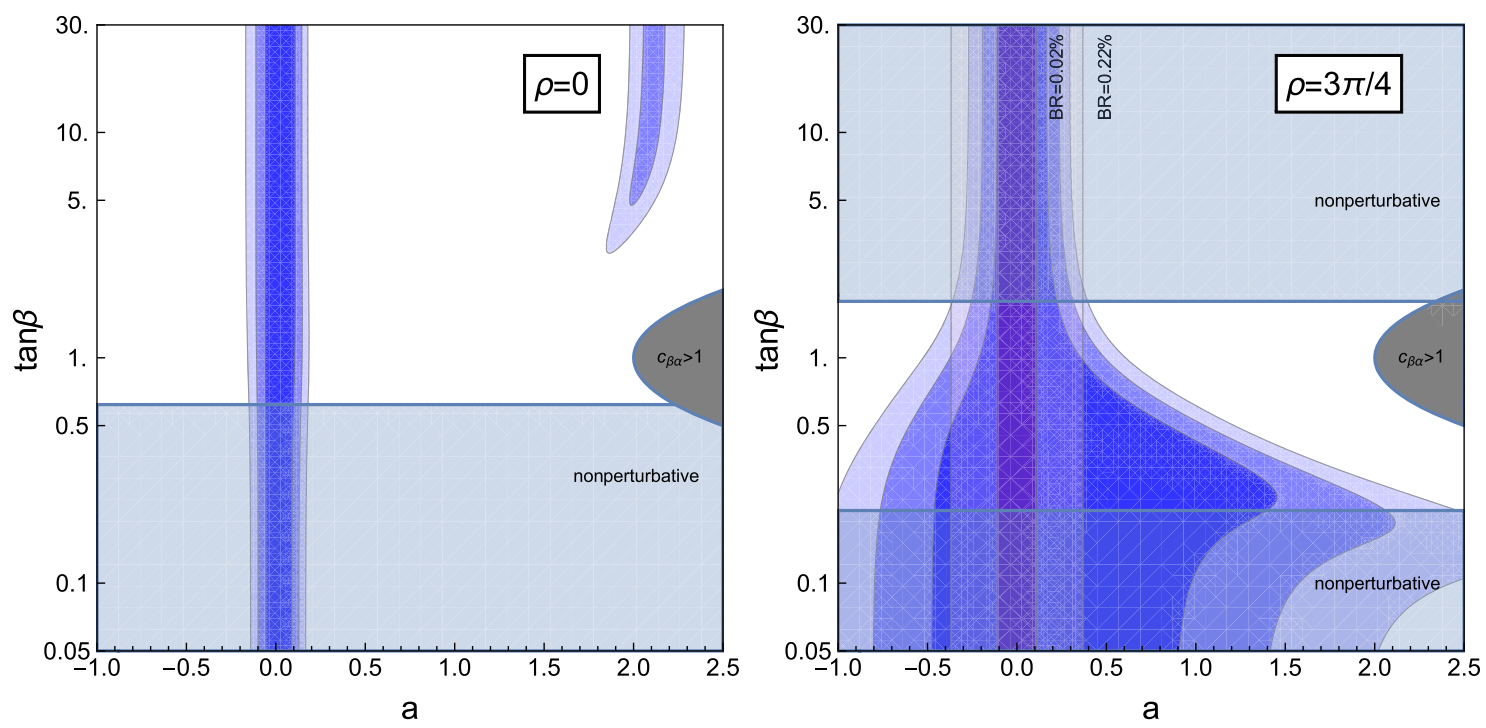

FIG. 3. Allowed parameter space in the $a-\tan \beta$ plane. The left plot assumes $\rho=0$ (no mixing) while the right plot has $\rho=3 \pi / 4$, where top-charm flavor mixing effects are almost maximal. Even though the mixing effect is maximized, it is difficult to realize a branching ratio of $0.22 \%$ for the $t \rightarrow c h$ decay in the large $\tan \beta$ case.

observables. For a fixed $\tan \beta$ value, the blue regions always become broader along the $a$ direction when $\rho$ is turned on and broadest around $\pi / 2$ to $3 \pi / 4$. This tendency is stronger when $\tan \beta$ is smaller. This can be understood as follows from the fact that the latest Higgs data are essentially consistent with the SM expectations, and a large deviation in the signal strengths disfavored. The gauge boson couplings to the Higgs are consistent with the SM, and it forces $\sin (\beta-\alpha) \simeq 1$ and $\cos (\beta-\alpha)$ to be small. Thus, each signal strength $\mu_{i}$ from the $g g H$-initiated mode is roughly proportional to $\left(\xi_{t} / \xi_{b}\right)^{2}$, taking the fact that the total width is mainly controlled by the bottom Yukawa coupling. From the expression Eq. (15), we see $d\left(\xi_{t} / \xi_{b}\right) \simeq$ $(1+\cos \rho)(\tan \beta+\cot \beta) d \cos (\beta-\alpha)$, and this part of the corrections becomes milder with nonzero $\rho$. Moreover, for small $\tan \beta$, a larger $a$ is realized due to large $\cot \beta$ whereas it does not initiate large effects on $\xi_{b}$ or on the total width.

There is also a so-called "wrong-sign Yukawa" solution with $\xi_{t}=1$ and $\xi_{i}=-1$ ( $i$ : other than the top) for large $\tan \beta$. In this case, the Yukawa couplings of quarks other than the top quark have an opposite sign to their SM values [46], achieved by having $\tan \beta \cos (\beta-\alpha) \simeq-2$ but $(1-\cos \rho) \tan \beta \cos (\beta-\alpha) \sim 0$. One can understand why the corresponding solution does not exist for small $\tan \beta$ nor for large $\rho$ from this expression. For $\tan \beta=10$, there remains viable parameter space at $a \sim 2.2$ as well as normal $a \sim 0$ region for small $\rho$ although the wrong-sign region is compatible with the Higgs data at the $2 \sigma$ level, as seen in the right lower plot of Fig. 2.

The FCNC top rare decay process $t \rightarrow c h$ can put useful constraints on the parameter space as well. Such FCNC effects are proportional to $a^{2} \sin ^{2} \rho$. The nominal FCNC branching ratios of $\leq 0.22 \%$ and $\leq 0.02 \%$ are depicted in the plots by the pink and red regions, respectively. If the nominal size of the signature top decay turns out to be a real signal, we can exclude the $\rho \sim 0$ region allowed by the current Higgs signal strength data.

In Fig. 2, we also superimpose the requirement of perturbativity in the Yukawa couplings. For $\tan \beta>1$ $(\tan \beta<1)$, larger (smaller) $\rho$ regions are ruled out, as indicated by the shaded light-gray region. There is no such constraint for the $\tan \beta=1$ case. However, we have a darkgray region ruled out by $c_{\beta \alpha}>1$ in this case.

Figure 3 shows the allowed parameter space in the $(a, \tan \beta)$ plane of our model, as constrained by the current Higgs data when the flavor mixing effect is switched off $(\rho=0)$ in the left plot and switched on almost maximally $(\rho=3 \pi / 4)$ in the right plot. Again, the second branch of solution in the left plot appears only when $\tan \beta$ is sufficiently large. At 95\% C.L., the branch of $a \sim 0$ is constrained to have $|a| \lesssim 0.2$ when the mixing effect is switched off. In the case where the mixing effect is larger, the allowed parameter region is slightly relaxed but still constrained to be $|a| \lesssim 0.3$ for $\tan \beta>1$. On the other hand, for the smaller $\tan \beta$ region, the allowed $a$ region is significantly extended. Furthermore, one can see in the right plot that even with $\rho=3 \pi / 4$, which provides almost maximized FCNCs in $\rho$, larger $\tan \beta$ is incompatible with $\operatorname{BR}(t \rightarrow c h)=0.22 \%$. Therefore, we can conclude that there exists an upper bound on $\tan \beta$ for the nominal value of $\operatorname{BR}(t \rightarrow c h)=0.22 \%{ }^{1}$ Larger $\tan \beta$ with larger mixing $\rho$ is also disfavored by the perturbativity requirement.

\footnotetext{
${ }^{1}$ There is an exception of the wrong Yukawa solution with a sufficiently large $\tan \beta$, although the parameters are compatible with the Higgs data at the $2 \sigma$ level.
} 


\section{FURTHER TESTS FOR THE MODEL}

Once we observe a sufficient number of $t \rightarrow c h$ events, it will be possible to check the chiral nature of the Higgs flavor-changing couplings as predicted in the model: the charm quark in the decay product should be right handed. The spin analyzing power $\kappa_{i}$ of particle $i$ in the decay product is defined as

$$
\frac{1}{\Gamma_{i}} \frac{d \Gamma_{i}}{d \cos \theta_{i}}=\frac{1}{2}\left(1+\kappa_{i} P \cos \theta_{i}\right)
$$

where $P$ is the polarization of the mother particle along a specific direction, called the polarization axis, $\Gamma_{i}$ is the partial decay width of the mode containing particle $i$, and $\theta_{i}$ is the polar angle of particle $i$ with respect to the polarization axis. The spin analyzing power of the charged lepton, $\kappa_{\ell^{+}}$, from the usual top decay $t \rightarrow b \ell^{+} \nu$ is known to have the largest value +1 at leading order [47]. We denote the spin analyzing power for the antitop quark decay by $\bar{\kappa}$ and note that $\bar{\kappa}_{\bar{f}}=-\kappa_{f}$ assuming $C P$ invariance. Our model predicts $d \Gamma_{t \rightarrow c h} / d \cos \theta \propto 1+\cos \theta$, and the charm quark and the Higgs boson have the spin analyzing powers $\kappa_{c}=+1$ and $\kappa_{h}=-1$, respectively. Once we know the original top spin direction, we can readily determine $\kappa_{c}$ and $\kappa_{h}$ in the $t \rightarrow c h$ decay.

We have discussed in Ref. [14] the possibility of determining the chirality structure using the top spin correlation in $t \bar{t}$ production at the LHC $[48,49]$ as the top quarks in top pair production are not polarized and not directly usable. The differential cross section in the double theta distribution is given by

$$
\frac{1}{\sigma} \frac{d \sigma}{d \cos \theta_{i} d \cos \theta_{j}}=\frac{1}{4}\left(1+A_{\mathrm{hel}} \kappa_{i} \bar{\kappa}_{j} \cos \theta_{i} \cos \theta_{j}\right),
$$

where $\theta_{i, j}$ are defined in the rest frame of the top and antitop quarks, respectively, and the $t \bar{t}$ spin asymmetry defined in the helicity basis [50] is

$A_{\text {hel }} \equiv \frac{N\left(t_{\uparrow} \bar{t}_{\uparrow}\right)+N\left(t_{\downarrow} \bar{t}_{\downarrow}\right)-N\left(t_{\uparrow} \bar{t}_{\downarrow}\right)-N\left(t_{\downarrow} \bar{t}_{\uparrow}\right)}{N\left(t_{\uparrow} \bar{t}_{\uparrow}\right)+N\left(t_{\downarrow} \bar{t}_{\downarrow}\right)+N\left(t_{\uparrow} \bar{t}_{\downarrow}\right)+N\left(t_{\downarrow} \bar{t}_{\uparrow}\right)} \sim 0.35$

at the LHC.

For a rough estimate of the required number of events to determine $\kappa_{h}$ (or $\kappa_{c}$ ) by measuring the angular distribution of $i=\ell^{+}, j=h$ and the corresponding antiparticle case, we have also introduced a simpler observable out of the above-mentioned observables as

$$
\begin{aligned}
A_{\ell h} & \equiv \frac{N\left(\cos \theta_{\ell} \cos \theta_{h}>0\right)-N\left(\cos \theta_{\ell} \cos \theta_{h}<0\right)}{N\left(\cos \theta_{\ell} \cos \theta_{h}>0\right)+N\left(\cos \theta_{\ell} \cos \theta_{h}<0\right)} \\
& =\frac{A_{\mathrm{hel}} \kappa_{\ell} \bar{\kappa}_{h}}{4} \sim 0.088 \bar{\kappa}_{h} .
\end{aligned}
$$

To confirm that $\kappa_{h} \sim-1$, we have to measure a positive $A_{\ell h}$ at a precision better than 0.088. The statistical uncertainty on $A_{\ell h}$ is then given by

$$
\Delta A_{\ell h} \simeq \Delta N / N \simeq 1 / \sqrt{N}<0.088,
$$

implying that we need at least $\sim 130$ signal events to confirm the decay distribution structure at the $1 \sigma$ level. As we expect $3 \times 10^{9}$ top pair events using $\sigma(t \bar{t}) \sim 1 \mathrm{nb}$ and an integrated luminosity of $3000 \mathrm{fb}^{-1}$ at the $14 \mathrm{TeV}$ LHC, it provides $\sim 10^{7} t \rightarrow c h$ events for the nominal branching ratio of $0.22 \%$. Even considering only the cleanest mode $h \rightarrow \gamma \gamma$, we still expect $\sim 5000$ events after multiplying $\mathrm{BR}(h \rightarrow \gamma \gamma) \sim 2.3 \times 10^{-3}$ and the leptonic decay branching ratio of the top quark. Besides, the $h \rightarrow b \bar{b}$ mode can be incorporated to enhance the signal significance [51]. Therefore, with the assumption of the nominal branching ratio, one can easily determine the chirality structure of the flavor-changing Higgs coupling at the ultimate integrated luminosity in LHC run-III.

Recently, the high-energy upgrade of the LHC (HE-LHC) is more seriously discussed and its target center of energy and integrated luminosity are realistically decided as $27 \mathrm{TeV}$ and $12 \mathrm{fb}^{-1}$ [52]. At $\sqrt{s}=27 \mathrm{TeV}$, the $t \bar{t}$ cross section reaches $3.7 \mathrm{nb}$ computed by hathor 2.0 [53], therefore, we expect that the sensitivity on the branching ratio improves below $10^{-5}$ due to 15 times as many as $t \bar{t}$ events.

\section{CONCLUSION}

In this paper, we consider the current status of the topspecific variant axion model in light of the latest Higgs data and a slight excess in the $t \rightarrow c h$ decay. This model is well motivated to solve the strong $C P$ and domain wall problems. As the top FCNC decay is one of the generic predictions of the model, we discuss whether it is possible to have a sizable $\mathrm{BR}(t \rightarrow c h)$ under various theoretical and phenomenological constraints. We find that to realize a rather large branching ratio of $0.22 \%$ in this model, $\tan \beta \sim 1$ or smaller is preferred by the current Higgs data. Such a preference is also supported by the perturbativity requirement on the Yukawa couplings. In other words, what we show is that although the Higgs signal strength data are essentially the same as the SM predictions, our model can readily accommodate a sizable $\mathrm{BR}(t \rightarrow c h)$ without conflicts with the Higgs data as long as $\tan \beta \lesssim 1$.

The $h-t-c$ vertex has a specific chirality structure according to the model. We therefore propose to measure this characteristic feature as an essential step toward verifying the model. We show that this can be done by measuring the spin correlation in the top pair production through the $t \rightarrow c h$ mode and estimated that the required sensitivity for confirming the model can be achieved by the end of LHC run-III, assuming $\operatorname{BR}(t \rightarrow c h)=0.22 \%$ is realized in nature. 


\section{ACKNOWLEDGMENTS}

This research was supported in part by the Ministry of Science and Technology of Taiwan under Grant No. NSC 100-2628-M-008-003-MY4 (C.-W. C) and in part by the Grants-in-Aid for Scientific Research from the Ministry of Education, Culture, Sports, Science, and Technology (MEXT), Japan No. 26104009 and
Grant-in-Aid No. 26287039 from the Japan Society for the Promotion of Science (JSPS) (T. T. Y.); JSPS Grant-in-Aid for Scientific Research Nos. JP16H03991, JP16H02176, JP17H05399 (M. T.); and the World Premier International Research Center Initiative (WPI Initiative), MEXT, Japan (H. F., M. T. and T. T. Y.).
[1] R. D. Peccei and H. R. Quinn, Phys. Rev. Lett. 38, 1440 (1977).

[2] S. Weinberg, Phys. Rev. Lett. 40, 223 (1978).

[3] F. Wilczek, Phys. Rev. Lett. 40, 279 (1978).

[4] K. A. Olive et al. (Particle Data Group Collaboration), Chin. Phys. C 38, 090001 (2014).

[5] L. F. Abbott and P. Sikivie, Phys. Lett. 120B, 133 (1983).

[6] J. Preskill, M. B. Wise, and F. Wilczek, Phys. Lett. 120B, 127 (1983).

[7] M. Dine and W. Fischler, Phys. Lett. 120B, 137 (1983).

[8] P. A. R. Ade et al. (Planck Collaboration), Astron. Astrophys. 594, A13 (2016).

[9] M. Kawasaki, K. Saikawa, and T. Sekiguchi, Phys. Rev. D 91, 065014 (2015).

[10] R. D. Peccei, T. T. Wu, and T. Yanagida, Phys. Lett. B 172, 435 (1986).

[11] L. M. Krauss and F. Wilczek, Phys. Lett. B 173, 189 (1986).

[12] C. Q. Geng and J. N. Ng, Phys. Rev. D 41, 3848 (1990).

[13] C. R. Chen, P. H. Frampton, F. Takahashi, and T. T. Yanagida, J. High Energy Phys. 06 (2010) 059.

[14] C. W. Chiang, H. Fukuda, M. Takeuchi, and T. T. Yanagida, J. High Energy Phys. 11 (2015) 057.

[15] V. Khachatryan et al. (CMS Collaboration), Phys. Lett. B 749, 337 (2015).

[16] G. Aad et al. (ATLAS Collaboration), J. High Energy Phys. 11 (2015) 211.

[17] CMS Collaboration, Report No. CMS-PAS-HIG-17-001.

[18] G. Aad et al. (ATLAS Collaboration), J. High Energy Phys. 12 (2015) 061.

[19] V. Khachatryan et al. (CMS Collaboration), J. High Energy Phys. 02 (2017) 079.

[20] M. Aaboud et al. (ATLAS Collaboration), J. High Energy Phys. 10 (2017) 129.

[21] ATLAS Collaboration, Report No. ATL-PHYS-PUB-2013012.

[22] K. Agashe et al. (Top Quark Working Group Collaboration), arXiv:1311.2028.

[23] ATLAS Collaboration, arXiv:1307.7292.

[24] S. Davidson and H. E. Haber, Phys. Rev. D 72, 035004 (2005).

[25] L. Lavoura and J. P. Silva, Phys. Rev. D 50, 4619 (1994).

[26] R. Harnik, J. Kopp, and J. Zupan, J. High Energy Phys. 03 (2013) 026.
[27] M. Bona et al. (UTfit Collaboration), J. High Energy Phys. 03 (2008) 049.

[28] M. Gorbahn and U. Haisch, J. High Energy Phys. 06 (2014) 033.

[29] G. C. Branco, P. M. Ferreira, L. Lavoura, M. N. Rebelo, M. Sher, and J. P. Silva, Phys. Rep. 516, 1 (2012).

[30] G. Aad et al. (ATLAS and CMS Collaborations), J. High Energy Phys. 08 (2016) 045.

[31] ATLAS Collaboration, Report No. ATLAS-CONF-2017045.

[32] ATLAS Collaboration, Report No. ATLAS-CONF-2017043.

[33] CMS Collaboration, Report No. CMS-PAS-HIG-16-040.

[34] CMS Collaboration, Report No. CMS-PAS-HIG-16-041.

[35] CMS Collaboration, Report No. CMS-PAS-HIG-16-021.

[36] A. M. Sirunyan et al. (CMS Collaboration), arXiv:1708 .00373 .

[37] CMS Collaboration, Report No. CMS-PAS-HIG-17-010.

[38] ATLAS Collaboration, Report No. ATLAS-CONF-2016112.

[39] ATLAS Collaboration, Report No. ATLAS-CONF-2016063.

[40] CMS Collaboration, Report No. CMS-PAS-HIG-16-003.

[41] ATLAS Collaboration, Report No. ATLAS-CONF-2017041.

[42] ATLAS Collaboration, Report No. ATLAS-CONF-2016068.

[43] CMS Collaboration, Report No. CMS-PAS-HIG-17-004.

[44] CMS Collaboration, Report No. CMS-PAS-HIG-17-003.

[45] CMS Collaboration, Report No. CMS-PAS-HIG-16-038.

[46] P. M. Ferreira, J. F. Gunion, H. E. Haber, and R. Santos, Phys. Rev. D 89, 115003 (2014).

[47] W. Bernreuther, J. Phys. G 35, 083001 (2008).

[48] G. Aad et al. (ATLAS Collaboration), Phys. Rev. Lett. 114, 142001 (2015).

[49] CMS Collaboration, Report No. CMS-PAS-TOP-13-015.

[50] W. Bernreuther, A. Brandenburg, Z. G. Si, and P. Uwer, Nucl. Phys. B690, 81 (2004).

[51] J. A. Aguilar-Saavedra, Acta Phys. Pol. B 35, 2695 (2004).

[52] https://indico.cern.ch/event/647676/contributions/2721141/ attachments/1548876/2433767/HE-LHC-Machine-2017.pdf.

[53] M. Aliev, H. Lacker, U. Langenfeld, S. Moch, P. Uwer, and M. Wiedermann, Comput. Phys. Commun. 182, 1034 (2011). 